\title{
SISTEMATIZACIÓN DE LA DIMENSIÓN AMBIENTAL EN LA UNIVERSIDAD DE CALDAS COMO UN MEDIO PARA GENERAR UN SISTEMA DE GESTIÓN AMBIENTAL DE LA INSTITUCIÓN
}

Ginés Fernando Ramírez Benavides (1)

Luz Elena Sepúlveda Gallego (2)

Manizales, 2007-11-15 (Rev. 2007-12-19)

\section{RESUMEN}

Esta investigación responde a un proceso de sistematización, mediante análisis documental, de la inclusión de la dimensión ambiental en los documentos administrativos, curriculares, investigativos y de proyección de la Universidad de Caldas, que tuvo las finalidades de elaborar una propuesta de política ambiental para la Universidad de Caldas y aportar insumos para generar un proceso reflexivo en torno al diseño y la implementación de un Sistema de Gestión Ambiental (SGA).

En términos generales, la Universidad de Caldas incluye alusiones a su compromiso ambiental en los documentos rectores de su quehacer institucional (PEI, Plan de Desarrollo, Planes de Acción y Políticas), pero carece de elementos articuladores que permitan concretar tal compromiso en su actuar misional. Por tal motivo, es importante que la Universidad de Caldas genere y aplique una Política Ambiental que permita la mencionada concreción a partir de un SGA y de los propósitos ambientales que le demandan, tanto la comunidad universitaria como su entorno.

\section{PALABRAS CLAVE}

Sistema de Gestión Ambiental, Ambiente, Institución de Educación Superior.

\section{SYSTEMATIZATION OF THE ENVIRONMENTAL DIMENSION IN THE UNIVERSIDAD DE CALDAS AS A MEANS OF GENERATING AN INSTITUTIONAL ENVIRONMENTAL MANAGEMENT SYSTEM}

\begin{abstract}
This research responds to a systematization process, by means of documentary analysis, the inclusion of the environmental dimension in administrative, curricular, investigative and projection documents of the Universidad de Caldas, with the purpose of elaborating a proposal on environmental policy for the Universidad de Caldas, and to contribute supplies to generate a reflective process on the design and implementation of an environmental management system.

In general terms, the Universidad de Caldas includes references to its environmental commitment in governing documents of its institutional tasks (PEI, Development Plan, Action Plans and policies), but lacks articulating elements that allow such commitment to be made in its mission and actions. For said reason, it is important that the Universidad de Caldas generates and applies an environmental policy that allows the mentioned concretion from an environmental management system and from the environmental intentions demanded by the university community as its surroundings.
\end{abstract}

\section{KEY WORDS}

Environmental management system, environment, higher education institution.

\section{INTRODUCCIÓN}

La Universidad de Caldas, como institución educativa de nivel superior, con amplio impacto y reconocimiento social y político, debe atender permanentemente los requerimientos que la sociedad en su conjunto le demande; en el cumplimiento de sus 
objetivos misionales y de sus funciones sustanciales genera no sólo impactos en el medio ambiente, sino también información, investigación científica y desarrollo tecnológico que debe contribuir a un desarrollo ambientalmente sustentable de la región y del país.

La pluralidad de pensamientos y de discursos que caracterizan los aspectos conceptuales en la Universidad, sumada a las dificultades de tipo administrativo y académico, han sido impedimentos claves en la determinación de las prioridades ambientales de la Universidad de Caldas y, por lo tanto, en el diseño y aplicación de un SGA.

Los acuerdos internacionales y la normativa nacional referente a la temática ambiental convierten en imperiosa la necesidad de dimensionar la perspectiva ambiental en el quehacer de cualquier institución y, más aún, si se trata de una institución de educación superior en tanto sus procesos investigativos, de proyección y de docencia deben partir de un conocimiento detallado de la temática ambiental con la finalidad de poder contribuir al logro de un ambiente sano, entendido en su perspectiva compleja, es decir, desde la imbricación de lo social y lo natural.

\section{OBJETIVOS Y PROPÓSITOS}

Esta investigación tuvo como objetivos: sistematizar la dinámica ambiental en investigación, proyección, docencia y administración de la Universidad de Caldas; elaborar una propuesta de política ambiental para la Universidad de Caldas; y aportar insumos para generar un proceso reflexivo en torno al diseño y la implementación de un SGA para la Universidad de Caldas.

Con el desarrollo de este proyecto se pretendió generar una dinámica reflexiva que articulara la administración como potencializadora de las funciones sustantivas de la Universidad (investigación, proyección y docencia) en la dimensión ambiental; además de generar procesos y espacios colectivos de reflexión y sistematización en torno a lo ambiental, para establecer las bases de construcción de un SGA.

\section{MATERIALES Y MÉTODO}

El método utilizado para analizar la información fue la revisión documental, mediante procesos de codificación y categorización. Para abordar el aspecto administrativo se estudiaron los siguientes documentos institucionales: Proyecto Educativo Institucional (PEI), Estatuto General, Plan de Desarrollo 2003-2007 con sus planes de acción correspondientes, resultados de la autoevaluación institucional, Sistema Integrado de Gestión y algunas de las solicitudes de informes realizados por la Contraloría General de la Nación. Para obtener la información referente a la función investigativa de la Universidad, se revisó el Acuerdo 19 de 2000 y los proyectos de investigación activos e inscritos en la Vicerrectoría de Investigaciones y Postgrados. Para abordar el aspecto proyectivo de la Universidad se analizó la Política de Proyección Universitaria y los proyectos correspondientes que los departamentos incluyeron en el sistema de información académica. Lo relacionado con el aspecto curricular se analizó en la Política Curricular, en el documento producto de la resignificación curricular de los programas y en las asignaturas constitutivas de los planes de estudio.

\section{RESULTADOS Y DISCUSIÓN}

\subsection{ASPECTOS ADMINISTRATIVOS}

Desde sus principios rectores, consagrados en el Proyecto Educativo Institucional (PEI), la Universidad de Caldas demuestra compromiso con el logro de un desarrollo sustentable, articulando en este propósito la generación, apropiación, difusión y aplicación de conocimientos, lo cual se aprecia claramente en su misión. De esta forma reconoce la importancia que demanda el análisis de lo ambiental para el desarrollo de la región del centro-occidente colombiano. La misión de la Universidad reza: "La Universidad de Caldas, en cumplimiento de la función social que corresponde a su naturaleza pública, tiene la misión de generar, apropiar, difundir y aplicar conocimientos, mediante procesos curriculares, investigativos y de proyección, para contribuir a formar integralmente ciudadanos útiles a la sociedad, aportar soluciones a los problemas regionales y nacionales y contribuir al desarrollo sustentable y a la integración del centro-occidente colombiano" (3) . De igual manera, en la visión institucional se hace alusión a la capacidad de la Universidad para proponer alternativas de acción en pro del desarrollo sustentable.

EI PEI está permeado por la perspectiva ambiental mediante alusiones explícitas al desarrollo sostenible y sustentable, al equilibrio ambiental, al conocimiento de las problemáticas regionales y nacionales, al estudio y conservación de los bienes 
naturales y culturales, a la conservación de un ambiente sano, al fomento de la educación y la cultura ecológica, a la educación ambiental en el área de formación general y al desarrollo de nuevos programas académicos directamente relacionados con lo ambiental.

Según el Estatuto General de la Universidad de Caldas, "los objetivos y el desarrollo de los campos de acción de la Universidad, estarán enmarcados en los aspectos sociales, económicos, políticos y culturales de su área de influencia y del país" lo cual, obviamente, considera la dimensión ambiental, a pesar de que no se hace explícitamente. Para el logro de esos objetivos y el desarrollo de los campos define las funciones de las diferentes instancias universitarias, en las cuales puede apreciarse de manera implícita, en unos casos, y explícita, en otros, la intencionalidad de involucrar lo ambiental en el quehacer universitario.

Para el logro de su misión, el Consejo Superior es el encargado de definir las políticas universitarias y la planeación de la Institución, así como vigilar que el funcionamiento de ésta esté acorde con las disposiciones legales, el Estatuto General y las políticas institucionales. En cuanto al Consejo Académico, una de sus funciones es "definir las políticas académicas de la Institución" y, por ende, definir el enfoque ambiental de los procesos formativos. Por su parte, la Secretaría General debe "asesorar jurídica y administrativamente al Rector y a las diferentes dependencias de la Universidad para que las normas, estatutos, reglamentos y demás actos jurídico-administrativos de la Institución estén dentro de un marco constitucional y legal y de conformidad con las normas vigentes", lo cual, de manera inevitable, debe incluir todo lo relacionado con la rendición de cuentas ante los organismos de control del Estado encargados de garantizar el derecho a un ambiente sano (4). Finalmente, la Vicerrectoría de Proyección Universitaria "tiene a su cargo, organizar y articular las relaciones de la Universidad a su interior, con el estado, con el sector público y privado, con las organizaciones no gubernamentales y con la sociedad civil con énfasis en la región centro - occidental del país y con una perspectiva nacional e internacional", lo cual, desde la perspectiva misional de la Universidad, obliga al compromiso con un modelo de desarrollo que se caracterice por su sustentabilidad ambiental.

Mención aparte merece la Dirección de Programa pues tiene como función "administrar el currículo de su programa, en consonancia con las Políticas Institucionales, el Plan de Desarrollo y las normas vigentes", entre las cuales se encuentra la Política Nacional de Educación Ambiental. Al respecto, uno de os objetivos de esta política es: "Formular estrategias que permitan incorporar la Educación Ambiental como eje transversal en los planes, programas y otros, que se generen tanto en el sector ambiental, como en el sector educativo y en general en la dinámica del Sistema Nacional Ambiental (SINA), desde el punto de vista no solamente conceptual (visión sistémica del ambiente y formación integral de los ciudadanos y ciudadanas del país) sino también desde las acciones de intervención de los diversos actores sociales, con competencias y responsabilidades en la problemática particular. Esto en el marco del mejoramiento de la calidad del ambiente, tanto local como regional y/o nacional, y por ende de la calidad de vida en el país".

Con la idea de dotar a la institución de un instrumento de gestión construido participativamente, que permitiera desarrollar lo expresado en el PEI, se configuró el Plan de Desarrollo 2003-2007 el cual, además, se justificó en aspectos como: monitorear la gestión y evaluar la efectividad, la pertinencia y el impacto institucional; enfrentar los riesgos derivados de la situación política, social, ecológica y económica del país; identificar los factores internos yexternos que influyen sobre el desarrollo de la institución; articular el desarrollo de la Universidad con planes, programas y proyectos locales, regionales y nacionales; y generar competencias sociales en términos de una mejor respuesta a la problemática social, económica y ambiental de la región de influencia ydel país.

Como elementos de los escenarios propios de la institución al momento de plantear el Plan de Desarrollo, se le dio importancia a las transformaciones sociales y ambientales que hacen necesaria la reconceptualización permanente de las relaciones de los seres humanos con su entorno; a los complejos procesos de globalización; a las crisis sociales, económicas y políticas que influyen en la vida institucional y amenazan su propia supervivencia; y a la mayor relevancia requerida de la investigación en la toma de decisiones concernientes al desarrollo político, económico, social y ambiental. Con un matiz claramente ambientalista, en el Plan de Desarrollo se definieron tres valores corporativos de la institución, a saber: respeto por las distintas formas de vida y la diversidad, solidaridad y respeto a la diferencia.

El Plan de Desarrollo 2003-2007 se centró en el logro y la definición de perspectivas programáticas para seis objetivos estratégicos. A continuación se presenta un análisis de cada uno de ellos. 
El objetivo estratégico número uno: "Diseñar, evaluar y aplicar un proceso de modernización, conversión y adaptación tecnológica, relacionado con los sistemas de información y comunicación, el sistema de laboratorios y la infraestructura física y dotación", se fundamenta exclusivamente en las actuales exigencias de modernización tecnológica, pero no contempla el hecho de la importancia del efecto positivo que sobre el ambiente debe tener tal modernización. Podría decirse que la perspectiva ambiental está incluida en dos de los programas de este objetivo estratégico: actualización y dotación tecnológica de equipos y laboratorios de la Universidad y desarrollo y mantenimiento físico y ambiental del campus y de la infraestructura en general.

En el objetivo estratégico número dos: "Consolidar una universidad socialmente competente mediante un sistema de proyección universitaria, entendido como apoyo a la gestión del desarrollo y a la promoción de la cultura", se puede develar el toque ambiental sólo en su fundamentación, cuando hace referencia a la necesidad de generar "respuestas adecuadas a las demandas sociales"; "contribuir de manera directa a la gestión del desarrollo" y a la "generación de alternativas a los problemas regionales y nacionales", de tal manera que la proyección adquiera "un carácter multidimensional mediante el cual se expresa la forma real del compromiso de la Universidad con la comunidad a la cual se debe". Se encuentra aquí el abordaje ambiental siempre y cuando se le reconozca como parte inherente a las demandas sociales y al desarrollo sostenible.

El objetivo estratégico número tres: "Generar una cultura investigativa que favorezca la producción, la difusión y la aplicación del conocimiento" presenta una clara fundamentación ambiental al reconocer que "la producción investigativa es hoy en día un insumo esencial para el desarrollo social, cultural y económico, ecológicamente sustentable de los individuos, las comunidades y las naciones" y que la Universidad es un "nicho donde se producen conocimientos y se forman investigadores, de cara al desarrollo e inspirados en ideales de democracia y conservación de la diversidad biótica y cultural en condiciones de intercomunicación planetaria".

Entendiendo lo ambiental como elemento inherente a lo social, el objetivo estratégico número cuatro: "Lograr altos niveles de calidad y efectividad académica en la oferta académica de pre y postgrado" tiene una clara tendencia ambiental pues considera que "existe una falta de adecuación cada vez más amplia, profunda y grave entre los saberes y las realidades. Los problemas son cada vez más pluridisciplinarios, transversales, globales y planetarios". La aceptación de la pluridisciplinariedad, más que en los problemas, en sus alternativas de solución, el reconocimiento de la transversalidad y de lo global y planetario, exigen la participación de los saberes ambientales.

En el objetivo estratégico número cinco: "Generar un clima organizacional que favorezca y estimule el cumplimiento de las labores académicas y administrativas y un sistema de bienestar que promueva el desarrollo integral de la comunidad universitaria" se presenta explícitamente el componente ambiental en tres de sus proyectos, a saber: prevención de la enfermedad, promoción de la salud y mejoramiento de la calidad de vida; mejoramiento de los ambientes físicos de trabajo; y generación de programas de seguridad industrial y salud ocupacional.

El objetivo estratégico número seis está formulado en estos términos: "Consolidar un sistema efectivo de planeación y de administración por procesos". Se parte del hecho de que la gestión ambiental hace parte de todos los procesos administrativos, de tal manera que, aunque no se menciona, se asume su existencia en los siguientes dos programas planteados como parte del objetivo: generación de indicadores de gestión internacional y adopción de un sistema de mejoramiento continuo soportado en procesos sistemáticos de evaluación y control en el conjunto de los procesos académicos y administrativos.

Dentro de la estrategia de planeación de la Universidad se decidió elaborar planes de acción anuales para la puesta en práctica del Plan de Desarrollo 2003-2007 de tal manera que respondieran a los objetivos estratégicos antes presentados.

En el plan de acción del año 2003 se le adicionó el término "desarrollo sustentable" al planteamiento del segundo objetivo estratégico, con lo cual se redimensiona el compromiso social de la Universidad hacia un fin específico. De las actividades que se propusieron para lograr el objetivo, dos son puntuales y se refieren a la gestión de los museos, las otras cuatro están presentadas de manera ambiciosa y poco tangible; no obstante, muestran un avance significativo en la intencionalidad institucional con respecto al desarrollo sostenible. Las actividades mencionadas son: apoyar institucionalmente a las personas desplazadas por la violencia y contribuir en la 
generación de alternativas frente al conflicto armado; avanzar en la generación de alternativas sostenibles y sustentables para el desarrollo local y regional; aportar a la generación de alternativas regionales en materia de políticas públicas en salud e iniciar un proceso en materia de gestión tecnológica que facilite el diseño y aplicación de tecnologías apropiadas en diferentes procesos productivos.

En este mismo plan de acción se definieron actividades relacionadas con la contextualización de la investigación mediada por el conocimiento de las necesidades y requerimientos de la región; actividades específicas para determinar la calidad de los ambientes laborales, entre ellas mediciones de ruido, radiación, iluminación, calor, frío y material particular.

Pese a que la fundamentación de los objetivos estratégicos cuatro y seis en el Plan de Desarrollo 2003-2007 tiene componentes ambientales, el desarrollo de los mismos en el plan de acción 2003 no siguió la misma línea.

Respecto a los asuntos ambientales, el plan de acción 2004 propuso la realización de actividades relacionadas con el reordenamiento de la planta física, la intervención de los laboratorios con miras a su acreditación y el diseño de un SGA que tenía como propósito "diseñar un sistema que evalúe, sistematice, direccione y operativice el manejo de las emisiones y los impactos ambientales de las actividades propias de cada área de la Universidad".

Es importante resaltar que el plan de acción 2004 por primera vez tiene en cuenta la gestión ambiental institucional y le da un carácter administrativo; no obstante, su formulación denota debilidad conceptual frente a las exigencias de un verdadero SGA y presenta algunas inconsistencias, pues el propósito reduce el sistema a la caracterización de impactos ambientales negativos y las actividades responden sólo parcialmente a la denominación del proyecto, inclusive se presentan actividades puntuales que no responden a ningún direccionamiento institucional.

Para el año 2005, el plan de acción se propuso como uno de sus objetivos "formular una política y definir lineamientos de gestión orientados a minimizar y prevenir los impactos ambientales generados por la Institución" expresado en las siguientes actividades: elaborar, socializar y adoptar la Política Ambiental para la Universidad de Caldas; implementar el plan de manejo de riesgo ambiental para la Universidad; realizar las intervenciones en el área de acuerdo con la priorización del comité de obras físicas; formular los indicadores de Gestión Ambiental para la Universidad y sistematizar la información respecto al tema ambiental.

De esta manera, se evidencian avances en cuanto a la concepción del deber ser de la gestión ambiental de la Universidad en tanto se asume como parte de un sistema de gestión reconocido institucionalmente mediante una política específica e instrumentalizado mediante un plan de manejo del riesgo, la priorización de intervenciones, la formulación de indicadores de gestión y la necesidad de sistematizar la información correspondiente.

De manera coherente con los proyectos del año 2004 relacionados con los laboratorios y el espacio físico, el plan de acción 2005 propuso entre sus actividades: autoevaluar los laboratorios de la Universidad con base en factores de acreditación institucional; formulación del Plan de mejoramiento de los laboratorios de la Universidad; intervención en el sistema de extracción de gases en los laboratorios y diseño y puesta en marcha de la estrategia para la certificación de los laboratorios institucionales que prestan servicios a la comunidad. (ISO 17025).

Respecto al objetivo estratégico número dos, el plan de acción 2005 propone actividades exclusivamente relacionadas con el centro de museos con una visión muy limitada del "desarrollo y promoción de la cultura". En el tratamiento de este objetivo estratégico se plantea una variante del mismo: "Consolidar una universidad socialmente competente mediante un sistema de proyección universitaria, entendido como apoyo a la gestión del desarrollo sustentable social, humano y económico". Visto así, el mencionado desarrollo sustentable adquiere una perspectiva antropocéntrica en tanto descarta la sustentabilidad ambiental, lo que va en contra de los valores definidos originalmente en el Plan de Desarrollo 2003-2007.

El plan de acción 2005 generó un nuevo objetivo estratégico: "Fortalecer un sistema de bienestar universitario orientado a generar el desarrollo integral de la comunidad universitaria". Para su logro propuso cinco actividades entre las que se resalta la quinta: "consolidar el programa para el fomento de la Cultura de la Salud en la universidad".

En el plan de acción 2006 persisten las actividades ambientales relacionadas con los laboratorios y el espacio físico; otras de las actividades propuestas fueron: elaborar la 
propuesta de regionalización; desarrollar proyectos de atención -asesoría e intervención- orientados a grupos poblacionales e institucionales de algunos municipios; fortalecer la participación de la universidad en el diseño, ejecución y seguimiento de las políticas públicas; definir en las subregiones, donde hace presencia la institución, una agenda común en torno a asuntos estratégicos, con la participación del Estado y las formas organizadas de la Sociedad Civil.

En cuanto al proyecto "Gestión ambiental", el plan de acción 2006 propuso como actividades: consolidar la implementación del plan de manejo ambiental en las distintas dependencias de la Universidad; divulgar entre la comunidad universitaria las actividades relacionadas con la protección del medio ambiente y elaborar la propuesta para el manejo de residuos sólidos y líquidos de las cafeterías de la Universidad.

Como cierre al proceso de seguimiento de los planes de acción constitutivos del Plan de Desarrollo 2003-2007, las propuestas relacionadas con lo ambiental del último plan de acción se presentarán agrupadas por objetivos estratégicos con sus respectivos proyectos.

Para el logro del objetivo estratégico "Diseñar, evaluar y aplicar un proceso de conversión tecnológica debidamente presupuestado, relacionado con el sistema de información y comunicación, el sistema de laboratorios y la infraestructura física" se propuso el desarrollo de los siguientes proyectos: Sistema de Información Institucional Integrado; Plan Institucional de Ordenamiento e Inversión Física Dirección de Planeación y Fortalecimiento de la Red Institucional de Laboratorios.

Pese a que el objetivo del proyecto "Sistema de Información Institucional Integrado" planteaba el desarrollo de "Sistemas de Información en la Universidad de Caldas, que permitan la optimización de la información de carácter administrativo y académico para el seguimiento, evaluación y toma de decisiones relacionada con la gestión institucional como soporte al proceso de aseguramiento de la calidad", su desarrollo no evidenció lo relacionado con la información de carácter ambiental. Las deficiencias en la existencia y confiabilidad de esta información son algunos de los elementos que le impiden a la institución ejercer gestión ambiental de manera acorde con la normativa nacional.

El proyecto "Plan Institucional de Ordenamiento e Inversión Física Dirección de Planeación" tuvo como objetivo "Elaborar el Plan de Ordenamiento Físico y definir lineamientos para la recuperación, mantenimiento, optimización y expansión de los espacios de la Universidad", pero su desarrollo se centró en continuar con las líneas de trabajo definidas en los anteriores planes de acción.

El proyecto "Fortalecimiento de la Red Institucional de Laboratorios" tuvo como objetivo "Consolidar la Red Institucional de Laboratorios mediante procesos encaminados al mejoramiento de la calidad".

Para el logro del objetivo estratégico "Consolidar una Universidad socialmente competente mediante un sistema de proyección universitaria, entendido como apoyo a la gestión de desarrollo sustentable social, humano y económico" se propusieron 11 proyectos en cuyo desarrollo no se hace explícito o evidente el componente ambiental, excepción hecha de la continuidad del proyecto de regionalización propuesto desde 2006.

Para el logro del objetivo estratégico "Generar una cultura investigativa que favorezca la producción, difusión y aplicación del conocimiento" se propusieron varios proyectos, entre los cuales se destacan, por su posible connotación ambiental, los siguientes: realizar una convocatoria de investigación aplicada a la solución de problemas asociados al sector productivo o social; fortalecer, en asocio con la Vicerrectoría de Proyección, el trabajo con la Fundación Universidad-Empresa enfocándolo a proyectos de investigación aplicada; elaborar el proyecto del Instituto de Biotecnología; revisar las fortalezas de investigación de la universidad para determinar las áreas promisorias y fomentar la creación y consolidación de comités de ética para la investigación y los postgrados.

Para el logro del objetivo estratégico "Consolidar un sistema efectivo de planeación y administración por procesos" se plantearon 12 proyectos, dos de los cuales tienen implicaciones ambientales. Bajo el objetivo "Contribuir al mejoramiento de la prestación de los servicios de aseo, seguridad, transporte y mantenimiento, y consolidar el Plan Unificado de Compras para lograr racionalizar de los recursos" se propuso "Elaborar un plan de mantenimiento de las diferentes sedes y diseñar estrategias para el mejoramiento del servicio" y "Ejecutar la campaña de aseo y medio ambiente" el cual tenía incluida la realización de "conferencias de Medio Ambiente". El hecho de que lo ambiental se explicite solamente en aspectos como los mencionados, permite suponer que existe un imaginario institucional respecto a la estrecha relación 
entre lo ambiental, lo ornamental y el aseo pues, como se ha mencionado en múltiples apartes, lo ambiental permea el quehacer institucional pero de manera implícita.

Bajo el objetivo "Desarrollar la política ambiental de la Universidad de Caldas encaminada a disminuir los riesgos ambientales y a fomentar la gestión ambiental entre la comunidad universitaria" se planteó el desarrollo de las siguientes actividades: consolidar la implementación del plan de manejo ambiental en las distintas dependencias de la Universidad; promover la participación activa de los miembros de la comunidad universitaria en las actividades relacionadas con la protección del Medio; implementar el plan de manejo de residuos sólidos las cafeterías de la Universidad; difundir los indicadores de gestión ambiental de la Universidad de Caldas; articular el proyecto de gestión ambiental de nivel central con el plan de trabajo del comité ambiental de la Universidad; implementar el plan de manejo de residuos sólidos en las oficinas de la Universidad y propiciar el uso adecuado de las áreas comunes y del campus de la Universidad.

En la presentación de los planes de acción se evidencian vacíos conceptuales respecto a las implicaciones ambientales del quehacer universitario tanto desde la perspectiva social como desde la biológica y la administrativa, ésta en lo atiente a la urgente, y aún no lograda, implementación de un SGA. Estos vacíos han llevado, inclusive, a la ausencia de una línea de continuidad en la definición de la responsabilidad institucional de los asuntos ambientales; en los diferentes planes de acción se responsabilizaron instancias como la Vicerrectoría de Proyección Universitaria, la Vicerrectoría Administrativa, la Oficina de Bienestar Universitario, Servicios Generales y el Comité Ambiental. Vale la pena aclarar que no es adecuado pretender que lo ambiental se maneje desde una sola instancia, en tanto su característica esencial es que debe permear el quehacer institucional; no obstante, sí debe estar claramente definida la instancia responsable de generar procesos de gestión ambiental de la Universidad.

A pesar de que poco a poco la gestión ambiental ha ido tomando lugar en los planes de acción mediante el proyecto específico de gestión ambiental, se evidencia una pérdida de protagonismo de la misma en el plan de acción 2007 y un escaso compromiso institucional desde el planteamiento de sus políticas. Para demostrarlo, basta con tener en cuenta que en los planes de acción se supone erróneamente la existencia de una política ambiental, un plan de manejo ambiental y unos indicadores de gestión ambiental y se reduce el actuar a unas actividades puntuales anticontaminación, poco impactantes, sin fundamentación epistemológica y tendientes a sobrevalorar el aspecto biologicista de lo ambiental.

Las actividades encaminadas al logro de los demás objetivos estratégicos y en especial del segundo, referido a la proyección universitaria, muestran que el ideal de un desarrollo sustentable presentado en el objetivo sigue siendo un concepto vacío en tanto se enuncia pero no se desarrolla.

En el marco del proceso de autoevaluación con fines de acreditación de alta calidad, la Universidad de Caldas se propuso incluir como factor la gestión ambiental desde cinco parámetros: política ambiental, sistema de gestión ambiental, formación ambiental, gestión de residuos y salud ocupacional. Respecto a la temática ambiental, el análisis del proceso concluyó en la necesidad de intervenir los siguientes problemas: carencia de cultura y conciencia institucional con respecto a la perspectiva ambiental, tanto en los aspectos conceptuales como de gestión; inadecuado manejo de residuos en todas las dependencias y diferentes espacios de convivencia universitaria; altos grados de contaminación auditiva y olfativa; deficiente conocimiento del quehacer ambiental en la Universidad; no se aplican los propósitos explicitados en el PEI referidos a promover la conservación de un ambiente sano y fomentar la educación y cultura ecológica en la Universidad; y desarticulación de la Universidad con la agenda ambiental regional. La acción de mejoramiento propuesta fue desarrollar una política ambiental de la Universidad de Caldas que estuviera encaminada a disminuir los riesgos ambientales y a fomentar la gestión ambiental entre la comunidad universitaria.

Para el año 2007 la Universidad, a través de su Oficina de Planeación, define la creación de un Sistema Integrado de Gestión (SIG) que busca interrelacionar el Sistema Institucional de Autoevaluación y Aseguramiento de la Calidad, el Sistema de Gestión Ambiental, el Sistema de Control Interno, el Sistema de Gestión de la Calidad, el Sistema de Salud y Seguridad ocupacional y el Sistema de Información Institucional.

Los objetivos específicos propuestos por el SIG son: generar una cultura de evaluación y mejoramiento continuo donde la autorregulación, el autocontrol y el autocuidado sean pilares fundamentales en la construcción de una Universidad socialmente responsable; construir un referente institucional que sirva de base para 
una toma de decisiones efectiva y un proceso de planificación unificado; generar una cultura de trabajo por procesos, donde el trabajo en equipo se convierta en la estructura básica por excelencia; mejorar el clima y el ambiente de trabajo a través de una comunicación clara, veraz y oportuna; fortalecer los procesos de capacitación y formación de todo el personal en dirección a la generación de una cultura del aseguramiento de la calidad académica y administrativa; y generar una cultura de servicio en todos los actores e instancias de la Universidad.

La construcción de un SGA debería basarse en unos objetivos similares en tanto debe contener elementos de evaluación y mejoramiento continuo, posibilidad efectiva de toma de decisiones con el apoyo de un trabajo en equipo y coordinado con los demás sistemas y definición de procesos que estandaricen y garanticen el cumplimiento de la normativa ambiental.

EI SIG contempla cinco estrategias que aplican perfectamente a un SGA, éstas son: participativa, educativa, de comunicación, de mercadeo y de coordinación.

La elaboración del SIG se basa en la implementación y seguimiento de cuatro tipos de procesos: estratégicos, misionales, de apoyo y de evaluación. La gestión ambiental, entendida desde una perspectiva propia del siglo XXI, debe reflejarse en el desarrollo de todos y cada uno de los procesos establecidos (5) pues el desarrollo de los saberes ambientales y las exigencias sociales, políticas y económicas del momento actual y futuro han demostrado la inseparabilidad de lo ambiental respecto a lo social, la prioridad de lo ambiental en la cotidianidad y la necesidad de reconocer y admitir que toda actividad humana implica una postura frente al medio ambiente.

Como ente estatal perteneciente al SINA que realiza actividades de diverso orden, la Universidad de Caldas está sujeta a la supervisión de control de sus responsabilidades ambientales por parte de la Contraloría General de la Nación y, claro está, desde sus decisiones autónomas tendientes al cumplimiento de su misión.

Desde al año 2002 la Contraloría General de la Nación inició su proceso de control sobre el cumplimiento de la normativa ambiental en la Universidad de Caldas. Inicialmente la Universidad se declaró no responsable de este cumplimiento dada sus funciones misionales; no obstante, el órgano de control insistió sobre sus hallazgos y empezó a generar glosas que implicaban un plan de mejoramiento institucional a partir de 2004.

A lo largo de estos cuatro años, los hallazgos de la Contraloría han hecho alusión al SGA, los diagnósticos ambientales, la contaminación y el incumplimiento de las normas nacionales, de los planes de acción institucionales y los planes de manejo ambiental de las granjas.

Como resultado de los hallazgos de 2004, la Universidad debió constituir un Comité de Gestión Ambiental sobre el cual recaía la responsabilidad de hacer frente a las demandas de la Contraloría. El Comité de Gestión Ambiental (6) asumió una postura más proactiva e impactante sobre el desempeño ambiental de la Universidad y propuso superar la función asignada para dar respuestas inmediatas por la discusión y establecimiento de políticas institucionales conducentes al establecimiento de un SGA que, como uno de sus resultados, podría satisfacer las demandas de la Contraloría.

En 2004 la Contraloría afirmó que en el marco de su diagnóstico "No se evidenciaron estudios ambientales que reflejen el impacto ambiental del total de las actividades que desarrolla la entidad". Como respuesta a esta deficiencia, la Universidad contrató los servicios de una Ingeniera de Saneamiento y Desarrollo Ambiental con la finalidad de que apoyara el diseño de un SGA que normatizara, direccionara y operativizara el control de emisiones e impactos ambientales en todas las áreas de la Universidad. En el informe final de ese trabajo se presenta un diagnóstico de los impactos negativos que genera la Universidad en sus diferentes dependencias.

Ante estos hallazgos y las exigencias de la Contraloría General de la Nación, la Universidad ha implementado algunas medidas conducentes a disminuir los impactos ambientales negativos con énfasis en los laboratorios, el centro editorial y las granjas.

Como se evidencia, el diagnóstico ambiental que actualmente posee la Universidad es cualitativo $y$, aunque de gran importancia, no es suficiente para dimensionar la gravedad de algunos asuntos normativos y misionales relacionados con una falta de gestión ambiental; por ello se hace indispensable la cuantificación de los impactos para que los correctivos aplicados puedan ser valorados.

En el año 2007, por primera vez la Contraloría hace referencia al incumplimiento de actividades consignadas en los planes de acción y de manejo ambiental, lo cual 
denota falta de compromiso institucional con los asuntos ambientales pues los mencionados planes fueron elaborados por integrantes de la comunidad universitaria y surtieron el trámite de aprobación requerido institucionalmente.

\subsection{PROCESOS FORMATIVOS}

La recientemente aprobada política curricular de la Universidad parte de la intencionalidad educativa y pedagógica del PEI y del proceso de implementación, desarrollo y evaluación de la reforma curricular implementada en el año 2002. En este sentido, la autoevaluación institucional mostró la necesidad de fortalecer estos desarrollos curriculares en el marco de sus resultados, los cuales involucran el factor gestión ambiental.

En términos generales, la política curricular brinda suficientes oportunidades y espacios para desarrollar óptimamente procesos educativo-ambientales siempre y cuando estos se entiendan desde su perspectiva holística, interdisciplinar y compleja articulando aspectos biológicos, sociales, políticos, económicos, éticos y estéticos, mediante una continua interpretación crítica de la realidad y el diálogo constructivo de saberes y actores.

Los enfoques del currículo se constituyen en una oportunidad de gran valor pues justamente se comparten con los ideales de lo educativo ambiental, específicamente en lo referente a que sea holístico (7), cultural (8), interdisciplinario (9), abierto (10) y orientado al desarrollo de competencias (11). Vale la pena resaltar que el enfoque cultural dado al currículo actúa como una herramienta que la institución educativa ofrece a la sociedad para garantizar las condiciones, las destrezas y los ideales que dan sentido y razón de ser, lo cual podría entenderse como un sustento de desarrollo enmarcado en el concepto de sostenibilidad.

Una de las características del currículo de la Universidad de Caldas es su pertinencia, la cual le garantiza ser permeable y estar abierto a "los avances del conocimiento y a las necesidades, características y retos derivados del contexto social, cultural, ambiental y económico del ámbito local en el cual se desarrolla y del escenario regional, nacional e internacional que lo determina".

En las definiciones adoptadas para programas de pre y postgrado no se evidencian elementos que permitan garantizar formación ambiental acorde con lo planteado desde el PEI; no obstante en el componente de formación general, obligatorio para todos los programas de pregrado, se define que éste estará constituido por "actividades académicas que se orienten al desarrollo de competencias básicas que apuntan a la formación política, ética, estética, filosófica y ambiental de los estudiantes". Es importante resaltar que en el componente de formación en ciencias, arte o filosofía se hace referencia al desarrollo del soporte epistemológico de los saberes científicos, humanísticos y artísticos lo cual, se esperaría, debería abordar el enfoque ambiental.

Respecto a los componentes de los planes curriculares, la política define como obligatoria la "Justificación del Programa" que "debe incluir la pertinencia del programa en función de las necesidades reales de formación en el país y en el centro-occidente colombiano...". La pertinencia de un Programa del siglo XXI inevitablemente requiere la demostración de su compromiso ambiental en tanto es ésta la perspectiva que hoy en día marca todos los caminos de desarrollo de las profesiones, especialmente en lo referido a sus enfoques.

La política curricular considera que las mediaciones pedagógicas utilizadas en los procesos formativos deben favorecer la capacidad crítica y reflexiva, la resolución de problemas esperados e inesperados y el desarrollo de pensamiento, lo cual atañe directamente con los asuntos ambientales pues estos deben involucrarse en todos los procesos formativos, dado que deben ser integrales.

Entre las mediaciones pedagógicas, la política considera el uso de clases expositivas, talleres, seminarios de encuentro, salidas de campo, demostraciones, trabajos de observación, conversatorios, simulaciones, prácticas clínicas y medios virtuales. De la mano del desarrollo sostenible y de la reducción de los impactos negativos, se esperaría que el quehacer docente privilegiara el uso de mediaciones ambientalmente amigables, por ejemplo, las simulaciones y los medios virtuales. Actividades como las salidas de campo y los trabajos de observación deberían constituirse en momentos de sensibilización frente a la problemática ambiental.

Durante los años 2005 y 2006 la Vicerrectoría Académica coordinó el proceso de resignificación curricular que pretendía, entre otras cosas, generar el documento oficial de los planes curriculares de los programas académicos de pre y postgrado. 
Para efectos de la investigación se definió la utilidad de analizar el objeto de estudio, la investigación del Programa y la proyección del mismo bajo las categorías de interdisciplinariedad, enfoque científico y tecnológico, compromiso ético, perspectiva estética, enfoque social, enfoque ambiental explícito y compromiso con la transformación social; así como los objetivos del Programa, la identidad formativa y las competencias formativas, bajo las categorías de perspectiva o dimensión ambiental explícita, compromiso con lo social y la calidad de vida y transformación y desarrollo social.

Esta categorización está inspirada en la que, sobre formación ambiental, plantea la Política Nacional de Educación Ambiental; visto así, todas las categorías utilizadas forman parte de la dimensión ambiental. No obstante, para los fines específicos del trabajo, también se buscó identificar los Programas que de manera explícita hacen referencia al tema ambiental.

Los objetos de estudio de los Programas de la Facultad de Ciencias Agropecuarias carecen de evidencia de compromiso ético y de perspectiva estética, mientras que el enfoque científico y tecnológico es constante, el abordaje social y ambiental es apenas frecuente y el compromiso con la calidad de vida es escaso. En los objetivos y la identidad formativa del Programa, todos ellos abordan la temática ambiental y la mayoría, los aspectos relacionados con la calidad de vida y el desarrollo social. En cuanto a las competencias formativas, el abordaje ambiental y el compromiso con la calidad de vida son frecuentes mientras que lo relacionado con el desarrollo social es escaso. Tanto en investigación como en proyección prima el enfoque científico-tecnológico.

En la Facultad de Ciencia Exactas y Naturales la redacción del objeto de estudio contempla el énfasis científico-tecnológico de manera constante al igual que los planteamientos de la investigación y la extensión de los Programas; tanto en los objetivos, como en la identidad formativa, lo ambiental explícito es frecuente, siendo muy variable el comportamiento de las otras categorías.

En la Facultad de Ciencias para la Salud tanto los objetos de estudio como las intencionalidades investigativas y de proyección, muestran un direccionamiento científico-tecnológico y social; a pesar de que en los objetos de estudio los objetivos, las identidades formativas y las competencias formativas se presenta un compromiso con la calidad de vida, esto no se refleja en las intencionalidades de la investigación y la proyección. En todos los casos la alusión explícita a lo ambiental es escasa.

En los Programas de la Facultad de Artes y Humanidades no se hace referencia específica a lo ambiental ni al quehacer interdisciplinar; tampoco se alude al compromiso con la calidad de vida ni con aspectos éticos. Llama la atención que en el desarrollo de los objetivos de los Programas sí se contemple la temática de la calidad de vida.

En los aspectos relacionados con los objetos de estudio, la investigación y la proyección de los Programas de la Facultad de Ciencias Jurídicas y Sociales, se destaca el enfoque científico-tecnológico y el compromiso con lo social, lo cual también es evidente en los objetivos, la identidad formativa y las competencias formativas en tanto muestran de manera frecuente alusiones a la calidad de vida y al desarrollo social.

En la Facultad de Ingeniería el compromiso planteado es científico-tecnológico; en algunos Programas se mencionan aspectos éticos, sociales y ambientales.

La combinación de las categorías elegidas, como se mencionó anteriormente, pretende englobar la dimensión ambiental, por este motivo podría considerarse que las Facultades en las que con mayor frecuencia estaban presentes las categorías, son justamente las que más se acercan a una verdadera inclusión de la dimensión ambiental en su quehacer relativo a lo curricular. Las tres facultades con esta característica son la de Ciencias Agropecuarias, Ciencias para la Salud y Ciencias Jurídicas y Sociales. La Facultad que cuenta con menor frecuencia en cuanto a las categorías mencionadas es la de Artes y Humanidades.

Respecto a las categorías utilizadas, las que con mayor frecuencia se encontraron en el conjunto de facultades son: el enfoque científico-tecnológico y el aporte a la calidad de vida. Llama la atención la baja frecuencia de evidencias de interdisciplinariedad y la escasa alusión a los asuntos éticos, estéticos y ambientales, propiamente dichos.

Según la Política Nacional de Educación Ambiental, "atendiendo al carácter sistémico del ambiente, la Educación Ambiental debe ser considerada como el proceso que le permite al individuo comprender las relaciones de interdependencia con su entorno, a partir del conocimiento reflexivo y crítico de su realidad biofísica, social, política, 
económica y cultural para que, a partir de la apropiación de la realidad concreta, se puedan generar en él y en su comunidad actitudes de valoración y respeto por el ambiente. Estas actitudes, por supuesto, deben estar enmarcadas en criterios para el mejoramiento de la calidad de la vida y en una concepción de desarrollo sostenible, entendido éste como la relación adecuada entre medio ambiente y desarrollo, que satisfaga las necesidades de las generaciones presentes, asegurando el bienestar de las generaciones futuras". Desde esta perspectiva, una adecuada formación ambiental no se logra con la simple implementación de un curso referente a la misma, requiere ser planeada de tal manera que permee todo el proceso formativo del estudiante. A pesar de ello, siete Programas académicos incluyen en sus planes de estudio asignaturas específicas y aisladas para manejar los contenidos ambientales y educativo-ambientales, tales como: Ecología, Ecología y Educación Ambiental y Ecología Humana y Atención al Medio.

El desarrollo explícito de lo ambiental, relacionado con el objeto de estudio de los diferentes programas de pregrado, se realiza a través de múltiples actividades académicas. En doce Programas de pregrado no se evidencia ninguna actividad académica relacionada con lo ambiental, de manera genérica o profesionalizante.

En los postgrados no se evidencia un compromiso con la inclusión de la dimensión ambiental. Algunos de ellos abordan la temática por ser específica de su objeto de estudio.

\subsection{INVESTIGACIÓN}

El Acuerdo 19 de 2000 del Consejo Superior, que rige la investigación en la Universidad de Caldas, le da un carácter fundamental en la Universidad al concebirla como "todo proceso continuo y sistemático de generación del saber científico, técnico, humanístico o artístico; teniendo en cuenta que el concepto de saber se amplía más allá de su exclusiva significación como Ciencia para significar también el saber humanístico y artístico, cuyo rigor no depende de la exactitud matemática sobre el fenómeno que investiga sino del rigor conceptual y argumentativo de sus discusiones, del valor actual de las interpretaciones que generan y de la calidad de las obras que crean" y define como sus criterios: la pertinencia, la contextualización, la coherencia con la misión, visión y objetivos de la Universidad.

Desde esta perspectiva, si la investigación que fomenta la Universidad fuese realmente pertinente, contextualizada y coherente con lo planteado en el PEI, evidenciaría un marcado y explícito compromiso ambiental pues el contexto así lo exige. A manera de ejemplo, la Política de Investigación Ambiental de Colombia define la investigación ambiental como aquella "que se ocupa del estudio del entorno físico-biótico, de su relación con la estructura sociocultural, y de las dinámicas que tal relación conlleva. En tal sentido, la investigación y el conocimiento del ambiente son parte integral de la vida cotidiana de las poblaciones, y deben ser la base para la construcción de las relaciones armónicas con sus respectivos entornos" (12) . Se considera en esta Política de Estado que la investigación ambiental debe cubrir todos los campos del saber humano para lograr la generación de conocimiento que "coadyuve al logro del desarrollo sostenible" y ser transversal a todos los sectores productivos desde y hacia lo público y lo privado.

Como políticas institucionales de investigación, la institución adoptó el fomento de la cultura investigativa (13), el pluralismo (14) y la relevancia social (15) . Con esto se genera un espacio para la investigación ambiental pues fomenta la conformación de grupos inter y transdisciplinarios, la asunción de diferentes enfoques metodológicos y la búsqueda de solución de problemas que afectan la región y el país.

Desde el enfoque ambiental, merecen ser resaltados algunos de los objetivos que se propone la investigación de la Universidad de Caldas. Ellos son: proyectar como fortaleza la investigación para el estudio, el aprovechamiento, la conservación de la biodiversidad del medio ambiente social y natural y el uso sostenible de los recursos naturales de la región y el país; fomentar el trabajo académico interdisciplinario, transdisciplinario e interinstitucional, apoyado en procesos permanentes de investigación; y aplicar los resultados de la investigación en las actividades de proyección, docencia, extensión y servicios de la Universidad.

La Política de Investigación Ambiental colombiana reconoce como uno de sus nodos críticos la "falta de criterios y mecanismos claros de priorización que garanticen la pertinencia temática y regional, la relevancia y oportunidad de la investigación ambiental para la gestión ambiental", asunto que también caracteriza los procesos investigativos de la Universidad de Caldas. Específicamente en lo relacionado con la investigación ambiental, al igual que como se diagnostica en la Política Nacional de Investigación Ambiental, en la Universidad hay "poca valoración y reconocimiento 
institucional a la diversidad de modos de producción de conocimiento relevantes para el medio ambiente" y, más aún, poco reconocimiento de que se hace investigación ambiental.

De los 188 proyectos de investigación inscritos y activos en la Vicerrectoría de Investigaciones y Postgrados para el año 2007, sesenta y tres abordan asuntos ambientales desde las siguientes perspectivas: biológica-geológica (20), social (18), productiva (8), evaluación de impacto (6), Mixta (6) y epistemológica-ética (5). De estos datos se deduce que la tendencia de la investigación ambiental de la Universidad se da hacia los dos polos de la misma, es decir, hacia lo biológico y lo social; no obstante, también se cubren los otros aspectos posibles, aunque con un menor número de investigaciones.

La investigación de la Universidad de Caldas, en sí ambiental, debería explícitamente contribuir al cumplimiento del logro del objetivo general de la Política Nacional de Investigación Ambiental, el cual dice: "Fortalecer la capacidad nacional y regional que impulse la generación y utilización oportuna de conocimientos relevantes para el desarrollo sostenible, para lograr el mejoramiento la calidad ambiental y las condiciones de vida de la población colombiana, conforme a la diversidad natural y cultural del país y en armonía con la Política Nacional Ambiental".

\subsection{PROYECCIÓN}

"La proyección en la Universidad de Caldas tiene como misión integrar su desarrollo académico, científico, cultural, artístico, técnico y tecnológico con el entorno, propiciando la realización de procesos de interacción con los agentes sociales, con el fin de aportar a la solución de sus principales problemas, de participar en la formulación y construcción de políticas públicas y a contribuir en la transformación de la sociedad en una perspectiva de democratización y equidad social, en los ámbitos local, regional y nacional" (16) . A pesar de que la generalidad de los términos utilizados en la definición de esta misión permitiría la inclusión conceptual y operativa de los asuntos ambientales, la falta de un compromiso explícito con el mismo puede propiciar su no inclusión e, inclusive, su olvido.

La Proyección Universitaria de la Universidad de Caldas se encuentra reglamentada en su correspondiente política (17) . En el preámbulo de la Política se hace alusión implícita a lo ambiental en tanto se le otorgan criterios de regulación ética a la proyección, así como un papel protagónico al diálogo de saberes con la sociedad civil y compromisos con el fortalecimiento de la esfera de lo público en pos de la contribución a solución de los problemas de la región y la nación. Para los fines de esta investigación se considera que la bioética y la macrobioética (18) son tenidas en cuenta cuando se hace alusión a la ética.

Desde una mirada ambiental, tres de los considerandos de la Política de Proyección merecen ser resaltados en tanto se refieren a la construcción de políticas públicas, el establecimiento de un diálogo de saberes y la satisfacción de necesidades prioritarias; estos considerandos son: "que la proyección o la extensión hoy son dos conceptos homologables, considerados función sustantiva de la universidad, que independientemente de ser generadora o no de recursos, tienen como propósito el desarrollo de procesos continuos de interacción e integración con los agentes sociales y comunitarios, en orden de aportar a la solución de sus principales problemas, de participar en la formulación y construcción de políticas públicas y contribuir a la transformación de la sociedad desde una perspectiva de democratización y equidad social, local, regional y nacional, política y cultural, que es necesario reorientar la función de proyección en la Universidad hacia la satisfacción de un conjunto de necesidades prioritarias para el país, la región, la localidad, mediante la interacción y la integración con diversos actores sociales, urdiendo un tejido social que de consistencia a un proyecto institucional universitario de largo plazo; y que la interacción e integración de la Universidad con actores sociales permite un verdadero diálogo de saberes, logrando que las distintitas disciplinas nutran sus procesos de formación, proyección e investigación aportando a la construcción de capacidades individuales y sociales".

Si bien en la misión de la proyección no se explicita un compromiso ambiental de la Universidad en, al menos, dos de los principios orientadores sí se hace, específicamente en "pertinencia e impacto social" (19) y "responsabilidad social" (20) . En los principios orientadores "diálogo de saberes", "comunicación y relación con el sector productivo" y "corresponsabilidad", la mención es indirecta, al referirse a aspectos como la contextualización de las acciones con el medio, el compromiso con el sentido del bien público y el trabajo compartido y comprometido con el beneficio de las comunidades, los sectores y grupos poblacionales. 
Sólo uno de los objetivos de la proyección planteados en la Política podría verse relacionado con la inclusión de la dimensión ambiental: "Participar en los ámbitos nacional, regional y local, de manera proactiva, en la formulación y construcción de políticas públicas territoriales, así como de sus expresiones sectoriales en términos de la ciencia, la tecnología y las formas propias del arte y la cultura, concertando agendas, de tal modo que contribuyan al desarrollo social en sus diversas dimensiones", siempre y cuando una de esas "diversas dimensiones" sea la ambiental.

De manera consecuente con los principios de la Política, en los dos siguientes lineamientos de la misma se expresa la importancia de incluir lo ambiental en el quehacer de la proyección:

- Sostenibilidad: la universidad a través de las acciones de proyección, contribuirá a desarrollar en la comunidad la capacidad de pensar, construir y mantener a lo largo del tiempo un espacio común de convivencia, teniendo en cuenta las dinámicas y las interdependencias que existen entre el mundo natural y el entorno construido, así como los diferentes valores políticos, económicos, sociales, culturales y ambientales que la comunidad forja históricamente y la identifican.

- Gestión para la proyección: la universidad propiciará y facilitará la participación de su comunidad académica en diferentes escenarios culturales, tecnológicos, corporativos, empresariales, sociales y científicos, con el propósito de participar en la definición de políticas públicas de Estado, así como en la identificación de problemas a nivel local, regional y nacional, poner al servicio de éstos la capacidad humana, tecnológica, cultural y científica en la búsqueda de soluciones pertinentes a los contextos.

Finalmente, vale la pena resaltar que al definir la prestación de servicios de proyección como una de sus modalidades, se afirma que: "Por medio de los servicios la universidad pretende responder a las necesidades de su entorno; participa de manera efectiva en la solución de problemas; contribuye al desarrollo de una sociedad que amplía el vínculo que debe tener lo académico con lo público; fortalece, influye e interviene en las políticas de desarrollo social, político, ambiental y cultural, sin perder su autonomía Universitaria".

De los proyectos y las actividades de proyección vigentes para el segundo período académico del año 2007 abordan explícitamente lo ambiental las relacionadas con el seminario permanente del Departamento de Desarrollo Rural, el Observatorio de Conflictos Ambientales, el Jardín Botánico y la Revista Luna Azul. De las restantes 168 actividades de proyección podría considerarse que 24 incluyen, de alguna manera, aspectos ambientales; su categorización permite determinar que: 7 tienen enfoque biológico-geológico, todas ellas son conferencias aisladas o eventos programados; 8 se relacionan con evaluación de impacto ambiental, de ellas sólo dos son actividades permanentes y las demás son eventos programados; 2 tienden a un enfoque productivo, ambas son conferencias aisladas; 3 abordan lo social, todas ellas actividades permanentes y 4 tienen una perspectiva combinada, todas ellas actividades permanentes.

\section{CONCLUSIONES}

- En correspondencia con el Estatuto General, la inclusión de la dimensión ambiental en el quehacer institucional de la Universidad de Caldas es una responsabilidad compartida entre el Consejo Superior, la Rectoría, el Consejo Académico, las Vicerrectorías, la Secretaría General, las Decanaturas, las Direcciones de Departamento y Programa, la Jefatura de la Oficina de Planeación y la Jefatura de Control Interno.

- En el PEl y en el Plan de Desarrollo 2003-2007 se evidencia el compromiso institucional con lo relacionado al ambiente; no obstante, los planes de acción correspondientes no desarrollaron operativamente lo estipulado en el Plan de Desarrollo respecto a la temática ambiental; en la mayoría de los casos las actividades fueron puntuales y de poco impacto.

- La Universidad de Caldas fue pionera en la inclusión del factor ambiental como factor de autoevaluación con miras a la acreditación institucional; en el marco de esta autoevaluación se detectó la necesidad de intervenir los siguientes problemas: carencia de cultura y conciencia institucional con respecto a la perspectiva ambiental, tanto en los aspectos conceptuales como de gestión; inadecuado manejo de residuos en todas las dependencias y diferentes espacios de convivencia universitaria; altos grados de contaminación auditiva y olfativa; deficiente conocimiento del quehacer 
ambiental en la Universidad; no se aplican los propósitos explicitados en el PEI referidos a promover la conservación de un ambiente sano y fomentar la educación y cultura ecológica en la Universidad y desarticulación de la Universidad con la agenda ambiental regional.

- El quehacer institucional de la Universidad de Caldas genera impactos ambientales negativos, los cuales han sido parcialmente intervenidos.

- La recientemente aprobada Política Curricular de la Universidad de Caldas brinda suficientes oportunidades y espacios para el adecuado desarrollo de procesos educativo-ambientales; no obstante, entra en contradicción con la Política Nacional de Educación Ambiental al propiciar la creación de asignaturas específicas tendientes a garantizar la formación ambiental de los estudiantes cuando la misma debería permear todo el proceso formativo de los estudiantes.

- Desde el análisis documental de la resignificación curricular de los Programas, las Facultades que con mayor solvencia incluyen aspectos ambientales en los procesos formativos son: Ciencias Agropecuarias, Ciencias para la Salud y Ciencias Jurídicas y Sociales; por el contrario, la de menor evidencia es la Facultad de Artes y Humanidades.

- En los documentos de resignificación curricular de los Programas es escasa la alusión a la interdisciplinariedad, los aspectos éticos, lo relacionado con la estética y los asuntos ambientales.

- Sólo en la medida en que la investigación de la Universidad de Caldas sea realmente pertinente, contextualizada y coherente con lo planteado en el PEI, la misma evidenciaría un marcado y explícito compromiso ambiental. Actualmente, la perspectiva ambiental transversaliza al menos una tercera parte de los procesos investigativos que se realizan en la Universidad.

- Tal y como se afirma en la Política Nacional de Investigación Ambiental, en la Universidad de Caldas existe una desarticulación ente la investigación, la formación y la gestión ambiental.

- En el marco del quehacer misional de la Universidad, la Política de Proyección es la que de manera más adecuada incluye la perspectiva ambiental pues, además de hacerlo explícito, referencia permanentemente la contextualización de su quehacer, el diálogo de saberes, la participación en la definición de políticas públicas y el aporte a la construcción de sociedad; no obstante, y de manera contradictoria, las actividades y proyectos de proyección que abordan explícita y comprometidamente lo ambiental son escasas, aunque de un alto valor académico y social.

\section{RECOMENDACIONES}

- El Consejo Superior de la Universidad de Caldas debe definir como tarea prioritaria el establecimiento de una Política Ambiental de la Institución y, con base en ella, las instancias responsables deben propiciar la construcción de un SGA para la Universidad de Caldas que esté realmente articulado al SIG.

- La Universidad de Caldas debe buscar la asesoría que se requiera para garantizar un adecuado conocimiento por parte de los entes directivos de las obligaciones ambientales de la institución y sus correspondientes responsables.

- Los Consejos Superior y Académico deberían definir como una de las áreas estratégicas para el desarrollo misional de la institución el abordaje de la situación y la problemática ambiental.

- En el marco de la resignificación del PEI debe incluirse: La Universidad de Caldas, en el marco de sus funciones misionales, priorizará las actividades de docencia, extensión e investigación sobre aquellos problemas que impacten al medio ambiente y, por lo tanto, el sustento vital para todas las comunidades regionales, así como la compleja gama de interacciones ambientales que a nivel mundial comprometen la sobrevivencia del planeta a mediano plazo. Atendiendo a su naturaleza social, ha de considerar la relación humano-medio ambiente como el centro del conocimiento hacia donde debe dirigir los esfuerzos para la gestión de conocimientos de cuyo impacto se debe generar un ambiente equilibrado y sano. 
- El nuevo Plan de Desarrollo de la Universidad debe darle continuidad al compromiso institucional relacionado con el ambiente que se evidencia en el anterior; además, ese compromiso ambiental debe garantizase a partir de unos planes de acción que, evitando actividades puntuales, generen impactos ambientales positivos y reduzcan los impactos ambientales negativos derivados del quehacer institucional.

- En el marco del SIG, todos los procesos que para él se desarrollen deberían demostrar un abordaje desde la perspectiva ambiental, al menos en su parte conceptual; además, se hace necesario que en este sistema se incluyan los aspectos paisajístico y urbanístico.

- Con carácter de urgencia, se requiere la realización de un estudio técnico que de manera cuantitativa evalúe los impactos ambientales que genera la Universidad en el marco de su quehacer.

- La Vicerrectoría Académica debe establecer estrategias que garanticen una adecuada formación ambiental en todos los estudiantes, de tal manera que, superando las asignaturas aisladas y de bajo impacto, se propenda por la inclusión de la perspectiva ambiental en todas y cada una de las actividades académicas planteadas en los planes de estudio. De igual manera, la Vicerrectoría de Investigaciones y Postgrados debería propiciar la inclusión de la dimensión ambiental en los procesos formativos de los estudiantes de postgrado.

- La Universidad debe garantizar que todos los egresados de las Licenciaturas tengan la competencia para coordinar y desarrollar procesos educativo-ambientales enmarcados en la Política Nacional de Educación Ambiental.

- Replantear las prácticas didácticas, incluidas las prácticas académicas y los laboratorios, con la finalidad de disminuir en la mayor proporción posible los impactos ambientales negativos que las mismas puedan producir, específicamente en lo relacionado con el uso de reactivos, el desperdicio de recursos y el uso de sustancias tóxicas y peligrosas.

- Con base en la Política Nacional de Investigación Ambiental se recomienda impulsar programas de formación de investigadores ambientales a nivel de Maestría y Doctorado; fortalecer, ampliar y actualizar el componente ambiental de los programas de apoyo a grupos y centros de investigación; definir líneas temáticas, agendas y planes de acción a mediano y largo plazo para la investigación ambiental; y considerar los diversos modos de producción de conocimiento y las prioridades ambientales en el contexto nacional, regional e internacional.

- La Vicerrectoría de Proyección Universitaria debe comprometerse y apoyar iniciativas que propendan por el desarrollo social, político, económico y AMBIENTAL de las comunidades del área de influencia superando las actividades puntuales y aisladas de bajo impacto.

\section{BIBLIOGRAFÍA}

- colombia. CONGReso de la RepúblicA. Constitución Política de Colombia, 1991.

- - - CONGRESO DE LA REPÚBLICA. Ley 749 de 2002.

- ------. MINISTERIO DEL MEDIO AMBIENTE; CONSEJO NACIONAL AMBIENTAL; DEPARTAMENTO NACIONAL DE PLANEACIÓN; COLCIENCIAS. Política Nacional de Investigación Ambiental. Bogotá: El Ministerio, 2001.

- -------. MINISTERIOS DEL MEDIO AMBIENTE Y DE EDUCACIÓN NACIONAL. Política Nacional de Educación Ambiental. Bogotá: Los ministerios, 2002.

- GALLEGO, Carmenza. Propuesta sistema de gestión ambiental. Universidad de Caldas. 2005. Inédito.

- http://www.javeriana.edu.co/fear/ins_amb/sistema.htm [en línea] consulta: noviembre 10 de 2007.

- http://corambiental.udea.edu.co/comites/sga.htm [en línea] consulta: noviembre 10 de 2007.

- MAYR MALDONADO, Juan y otros. Bioética y medio ambiente. Bogotá: El bosque, 2000.

- MEJÍA BOTERO, Ximena. Identificación de impactos ambientales en la 
Universidad de Caldas, 2004. Inédito.

- UNIVERSIDAD DE CALDAS. CENTRO DE ADMISIONES Y REGISTRO ACADÉMICO. Planes de estudio de los programas académicos, 2006.

- ------. CENTRO DE ADMISIONES Y REGISTRO ACADÉMICO. Proyectos de investigación vigentes a 2007-2.

- -----.. CENTRO DE ADMISIONES Y REGISTRO ACADÉMICO. Proyectos y actividades de proyección vigentes a 2007-2.

- - CONSEJO ACADÉMICO. Política curricular de la Universidad de Caldas, 2007.

- --------. CONSEJO SUPERIOR. Acuerdo 064 de 1997.

- --------. CONSEJO SUPERIOR. Acuerdo 19 de 2000.

- ---------. CONSEJO SUPERIOR. Acuerdo 29 de 2002.

- -----. CONSEJO SUPERIOR. Acuerdo 08 de 2006.

- --------. DIRECCIÓN DE PLANEACIÓN. Sistema integrado de gestión, 2007. Inédito.

- ----------. Informe de Autoevaluación para la Acreditación Institucional de la Universidad de Caldas, 2006.

- -----. OFICINA DE CONTROL INTERNO. Plan de mejoramiento 2004.

- -----. OFICINA DE CONTROL INTERNO. Plan de mejoramiento 2005.

- ------. OFICINA DE CONTROL INTERNO. Plan de mejoramiento 2006.

- --------. OFICINA DE CONTROL INTERNO. Plan de mejoramiento 2007.

- ---------.. Plan de acción 2003.

- ---------. Plan de acción 2004

- -------.-. Plan de acción 2005.

- ------. Plan de acción 2006.

- -------. Plan de acción 2007.

- -------. Proyecto Educativo Institucional. Manizales: La Universidad, 1996.

- -----. VICERRECTORÍA ACADÉMICA. Resignificación curricular de los programas académicos, 2006.

\section{NOTAS}

1. Profesor asistente de la Universidad de Caldas, adscrito al Departamento de Salud Animal.

2. Profesora Titular de la Universidad de Caldas, adscrita al Departamento de Salud Pública.

3. UNIVERSIDAD DE CALDAS. Proyecto Educativo Institucional. Manizales: La Universidad, 1996. $p$.

4. Se refiere a la Contraloría General de la Nación, tal como se expresa en el artículo 53 del Estatuto General: "El control fiscal posterior o de gestión, será ejercido en la Universidad de Caldas por la Contraloría General de la República". Por funciones constitucionales otros entes de control son la Procuraduría y las Secretarías de Salud.

5. Los procesos estratégicos propuestos son: direccionamiento estratégico, planificación y sistema de información y comunicación; los misionales, docencia, investigación y proyección; los de apoyo, recursos académicos, bienestar, compras y contratación, asesoría jurídica y gestión de recursos; y los de evaluación, control y seguimiento y medición, análisis y mejora.

6. UNIVERSIDAD DE CALDAS. RECTORÍA. Resolución 00429 de junio 22 de 2004.

7. "Un currículo holístico, entendido como la relación integral entre el todo y sus partes, en donde cada parte contiene potencialmente al todo y la totalidad refleja la unidad íntima de sus partes; de tal manera que se genere una concepción sistémica de los procesos investigativos en donde el estudio de un área específica del conocimiento influye y se relaciona de manera estructural con la totalidad de conocimiento; se propicie una potencial relación interdisciplinar y transdisciplinar de cualquier área del conocimiento y de la investigación; se superen los saberes cerrados e independientes para ser comprendidos como saberes abiertos e interdependientes de la unidad curricular global; y se propicie la existencia de un diálogo de saberes, por parte de profesores y estudiantes de las distintas disciplinas y áreas curriculares, para completar una visión global de cualquier problema cognitivo específico". Política Curricular de la Universidad de Caldas.

8. "Un currículo cultural, entendido como un proyecto intencionado para transformar el orden cultural existente. Una propuesta curricular puede entenderse como el proyecto cultural de una sociedad pues la institución educativa se creó con el propósito de asegurar los medio para garantizar las condiciones, las destrezas y los ideales que dan sentido y razón de ser a una sociedad". Política Curricular de la Universidad de Caldas.

9. "Un currículo interdisciplinario, a partir de la convergencia de distintas disciplinas y perspectivas teóricas para el abordaje académico de todos los 
ejes problemáticos y temáticos que maneja la Universidad". Política Curricular de la Universidad de Caldas.

10. "Un currículo abierto, referido a un conjunto armónico, articulado, ordenado, dinámico y productivo de acciones docentes, investigativas y proyectivas que permitan entender la Universidad como un sistema que recibe, procesa, produce y transforma información en conocimientos científicos, artísticos y humanísticos". Política Curricular de la Universidad de Caldas.

11. "Un currículo orientado al desarrollo de competencias, a partir de la promoción de aprendizajes teórico-conceptuales, éticos, valorales, sociales, praxiológicos y procedimentales atendiendo a la naturaleza de los objetos y campos del conocimiento y los contextos del propio aprendizaje y de la realidad en la cual se ha desenvolver cada sujeto que aprende". Política Curricular de la Universidad de Caldas.

12. Ibíd. p. 6.

13. "Fomento de la Cultura Investigativa. La Institución debe fomentar la cultura investigativa, el espíritu científico, el desarrollo del conocimiento y la construcción de saberes en la comunidad universitaria, creando condiciones necesarias para la formación de investigadores y la conformación y consolidación de grupos de investigación en el marco de la disciplinariedad, interdisciplinariedad o transdisciplinariedad con proyección nacional e internacional". Acuerdo 019 de 2000 del Consejo Superior de la Universidad de Caldas.

14. "Pluralismo. Para las diferentes áreas del saber, la Universidad reconoce y promueve una concepción pluralista de la investigación, respetando diferentes enfoques teóricos, metodológicos y de resultados. La Universidad garantizará la libertad académica de los investigadores, en el marco de esta concepción pluralista, libertad que debe hacerse compatible con la esencia crítica, rigurosa, pública y abierta a la confrontación y discusión de la actividad investigativa". Acuerdo 019 de 2000 del Consejo Superior de la Universidad de Caldas.

15. "Relevancia Social. Sin desmedro de la investigación pura, y respetando el principio de libertad de cátedra, la Universidad promoverá procesos de investigación que puedan apoyar la solución de problemas que afectan la región y el país. Así mismo, se promoverán aquellos proyectos que contribuyan al desarrollo del pensamiento y la cultura". Acuerdo 019 de 2000 del Consejo Superior de la Universidad de Caldas.

16. UNIVERSIDAD DE CALDAS. CONSEJO SUPERIOR. Acuerdo 08 de 2006, artículo 1.

17. Ibíd.

18. "El término de macrobioética se asimila al sentido original de la bioética por lo menos en la idea que Potter quería asignarle como una referencia a los problemas de medio ambiente, los problemas demográficos, la unión con las ciencias médicas y biomédicas, y en general como él mismo lo sostiene al comienzo una ética al servicio de la supervivencia y de la buena vida en general". MAYR MALDONADO, Juan y otros. Bioética y medio ambiente. Bogotá: El bosque, 2000. p. 257.

19. "Pertinencia e impacto social: es la generación de impactos positivos en el medio social como en la comunidad universitaria, mediante la promoción y generación de proyectos y programas que se articulan con los contextos socioculturales, económicos, políticos y ambientales del entorno, contribuyendo a la solución de problemas que afecten la región y el país". Política de Proyección de la Universidad de Caldas.

20. "Responsabilidad social: es la respuesta ética a las demandas específicas del medio, mediante la creación de sinergia y retroalimentación permanente entre las líneas de acción institucional y el entorno, con el fin de atender y dar solución a diversas problemáticas que determinan las condiciones del bienestar social, humano y natural". Política de Proyección de la Universidad de Caldas. 